\title{
Surface-Based Vector Analysis Using Heat Equation Interpolation: A New Approach to Quantify Local Hippocampal Volume Changes
}

\author{
Hosung Kim, Pierre Besson, Olivier Colliot, Andrea Bernasconi, \\ and Neda Bernasconi \\ Department of Neurology and Brain Imaging Center, McGill University, Montreal \\ Neurological Institute and Hospital, Montreal, Quebec, Canada
}

\begin{abstract}
Analysis of surface-based displacement vectors using spherical harmonic description (SPHARM) localizes shape changes accurately. However, it does not allow differentiating volume variations from shifting and/or bending. We propose a new approach to quantify local volume changes by computing the surface-based Jacobian determinant. This measurement is computed on the displacement vector fields estimated by a heat equation interpolation on the displacement vectors produced by SPHARM. Data simulation showed that the surface-based Jacobian determinant enables accurate quantification of local volume changes without interference of shifting/bending. In patients with temporal lobe epilepsy and left hippocampal atrophy, SPHARM detected widespread inward deformation related to atrophy in the hippocampal head and body, and showed areas of mirrored inward/outward deformations mostly at the level of the hippocampal tail. In these areas, the surface-based Jacobian determinant showed atrophy. Our method facilitates the interpretation of SPHARM because it allows decomposing volume changes and shifting/bending. Furthermore, it provides a better delineation of the extent of hippocampal atrophy.
\end{abstract}

Keywords: brain morphometry, shape analysis, Jacobian analysis, heat equation, hippocampus.

\section{Introduction}

Surface shape models are objective methods to assess morphometric changes not evident in the measurement of the total volume. These methods have been successfully applied to quantify hippocampal shape in a variety of neurological and psychiatric disorders including epilepsy [1], Alzheimer's disease [2] and schizophrenia [3]. Among surface-based shape analysis methods, medial surface and axis models have been used to assess local variations in thickness [3]. These approaches produce symmetrical measurements on two facing surfaces. Alternatively, vertex-wise analyses of displacement vectors using surface registration techniques [1, 2] or the spherical harmonics description (SPHARM) [3, 4] localize asymmetrically shape changes. The signed surface normal component of the displacement vector is generally used to describe inward/outward deformations, which have been interpreted 
as the result of local volume changes [1]. However, this metric also captures positional differences between a given object and a template. Thus, it does not allow differentiating local volume variations from mirrored inward/outward deformations.

Deformation-based morphometry (DBM) enables the quantification of local volume changes without the interference of shifting/bending effects $[5,6]$. This technique produces 3D displacement vector fields between two non-linearly registered volume data. The Jacobian determinant map is computed on the vector fields and allows assessing local volume changes. DBM has been used to assess brain development [5] and atrophy in Alzheimer's disease [7].

The heat equation is a partial differential equation which describes the propagation process of heat over time [8]. Solving this equation from preset values on the boundary of a closed volume generates scalar fields within this volume. Therefore, this approach can be used to perform an isotropic and homogeneous interpolation.

Here, we propose an extension of SPHARM based on a heat equation interpolation method to estimate displacement vector fields inside a surface boundary. The vertexwise Jacobian determinant computed on the displacement vector fields will enable assessment of local volume changes without interference of shifting/bending.

\section{Methods}

Our method includes three main steps. First, using spherical harmonics description and point distribution models [4], we compute vertex-wise displacement vectors on a surface. Secondly, we interpolate the displacement vector fields within the surface boundary using the heat equation [8]. Finally, we compute the surface-based Jacobian determinant from the displacement vector fields to quantify local volume changes. We evaluate this method using simulation shapes and human hippocampi.

\subsection{Spherical Harmonics Description and Point Distribution Model}

Simulated objects/anatomical labels undergo a minimal smoothing operation. They are then converted to surface meshes for which a spherical parameterization (SPHARM) is computed using an area-preserving, distortion-minimizing mapping. Based on a uniform icosahedron-subdivision of the SPHARM, we obtain a point distribution model (PDM). SPHARM-PDM surfaces of each individual are rigidly aligned to a template constructed across subjects with respect to the centroid and the longitudinal axis of the $1^{\text {st }}$ order ellipsoid. Vertex-wise displacement vectors are calculated between each individual and the template. The surface normal component of this vector is analyzed to assess inner/outer deformations.

\subsection{Heat Equation}

The heat equation is a partial differential equation which describes the propagation process of heat in a given region over time [8]. For a function of three spatial variables $x, y, z$ and a time variable $t$, the heat equation is: 


$$
\frac{\partial u}{\partial t}=k\left(\frac{\partial^{2} u}{\partial x^{2}}+\frac{\partial^{2} u}{\partial y^{2}}+\frac{\partial^{2} u}{\partial z^{2}}\right)=k \Delta u
$$

where $k$ is a constant called conductivity and $\Delta$ is the Laplacian operator. From fixed scalar values on a boundary, this equation allows diffusing fields by minimizing the sum of gradient changes so that smooth fields are obtained at the equilibrium status. Using Laplacian operator, this equation can be applied to 3 orthogonal vector components $(\mathrm{x}, \mathrm{y}, \mathrm{z})$ without co-varying variables. Therefore, solving this equation with vectors on the surface $S$ of a closed volume as a boundary condition diffuses smooth vector fields within the volume. We used the displacement vectors $\mathbf{u}_{S}$ obtained in $\mathbf{2 . 1}$ as boundary condition. If a displacement vector at position $(x, y, z)$ in the volume domain is $\mathbf{u}=\left(\mathrm{u}_{x}, \mathrm{u}_{y}, \mathrm{u}_{z}\right)$, its variation at time $\mathrm{t}$ can be defined as:

$$
\frac{\partial \mathbf{u}}{\partial t}=\left(\frac{\partial u_{x}}{\partial t}, \frac{\partial u_{y}}{\partial t}, \frac{\partial u_{z}}{\partial t}\right)=\left(k \Delta u_{x}, k \Delta u_{y}, k \Delta u_{z}\right)
$$

Finally, the displacement vector fields in the volume inside $S$ are estimated by solving equation $(2)$ until reaching convergence $(\partial \mathbf{u} / \partial \mathrm{t} \rightarrow 0)$.

\subsection{Implementation on Discrete Space}

Let $\boldsymbol{\Omega}=\{(\mathrm{x}, \mathrm{y}, \mathrm{z}) \mid \mathrm{x}=0,1, \ldots, l ; \mathrm{y}=0,1, \ldots, m ; \mathrm{z}=0,1, \ldots, n\}$ be a discrete domain in 3D space. Consider a set of surface vertices $S=\left\{\mathbf{s}_{1}, \mathbf{s}_{2}, \ldots, \mathbf{s}_{M}\right\}$, on which the control lattice $\boldsymbol{\Omega}$ is overlaid (Figure 1-a). Then, let $\mathbf{u}_{\mathbf{s}}=\left\{\mathbf{u}_{\mathbf{S} 1}, \mathbf{u}_{\mathbf{s} 2}, \ldots \mathbf{u}_{\mathbf{S} M}\right\}$ be displacement vectors on $S$ calculated at the previous SPHARM-PDM stage. In 3D, we consider a surface point $\mathbf{s}_{\mathrm{i}}$ located within a unit-size cube enclosed by its adjacent 8 points on $\boldsymbol{\Omega}$. To compute the heat equation on $\boldsymbol{\Omega}$ with the boundary condition $\mathbf{u}_{\mathbf{s}}(\mathrm{t})=\mathbf{u}_{\mathbf{s}}, \mathbf{u}_{\mathbf{s i}}$ should be interpolated on domain $\boldsymbol{\Omega}$. Let $\mathbf{x}=(\mathrm{x}, \mathrm{y}, \mathrm{z})$ be one of these 8 points and $d\left(\mathbf{x}, \mathbf{s}_{\mathrm{i}}\right)$ be the Euclidean distance between $\mathbf{x}$ and $\mathbf{s}_{\mathrm{i}}$. Point $\mathbf{x}$ can be adjacent to one or more surface points (e.g., $\mathbf{S}_{\mathbf{i}-\mathbf{1}}, \mathbf{S}_{\mathbf{i}+\mathbf{1}}$ in Figure 1-b). We consider a general interpolation method at $\mathbf{x}$. Let $\mathrm{N}_{\mathrm{s}, \mathbf{x}}$ be the number of surface points adjacent to $\mathbf{x}$ and $\mathrm{A}_{\mathrm{d}, \mathrm{N}}$ be the average of distances between $\mathbf{x}$ and the adjacent surface points $s=\left\{\mathbf{s}_{\mathrm{i}}, \mathbf{s}_{\mathrm{i}+1}, \ldots, \mathbf{s}_{\mathrm{i}+\mathrm{N}-}\right.$ $1\}$. Then, we can compute $\mathbf{u}_{\mathbf{x}}$ by iterative search from $\mathbf{s}_{\mathrm{i}}$ to $\mathbf{s}_{\mathrm{i}+\mathrm{N}-1}$. When considering $\mathbf{s}_{\mathrm{i}}$, $\mathbf{u}_{\mathbf{x}}$ is determined as:

$$
\mathbf{u}_{\mathbf{x}}(t)=\mathbf{u}_{S_{i}}, A_{\mathrm{d}, 1}=d\left(\mathbf{x}, \mathbf{s}_{\mathrm{i}}\right)
$$

When $\mathrm{N}_{\mathrm{s}, \mathbf{x}}=\mathrm{N}, \mathbf{u}_{\mathbf{x}}$ is updated from the $\mathrm{N}-1^{\text {th }}$ computation $\left(\tilde{\mathbf{u}}_{\mathrm{x}}, \mathrm{A}_{\mathrm{d}, \mathrm{N}-1}\right)$ at $\mathbf{s}_{\mathrm{i}+\mathrm{N}-2}$ as:

$$
\begin{aligned}
& \mathbf{u}_{\mathbf{x}}=\frac{\mathbf{u}_{S_{i+N-1}} \cdot A_{d, N-1}+\widetilde{\mathbf{u}}_{\mathbf{x}} \cdot d\left(\mathbf{x}, \mathbf{s}_{i+N-1}\right)}{A_{d, N-1}+d\left(\mathbf{x}, \mathbf{s}_{i+N-1}\right)} \\
& A_{d, N-1}=\frac{A_{d, N-2} \cdot(N-2)+d\left(\mathbf{x}, \mathbf{s}_{i+N-2}\right)}{N-1}
\end{aligned}
$$




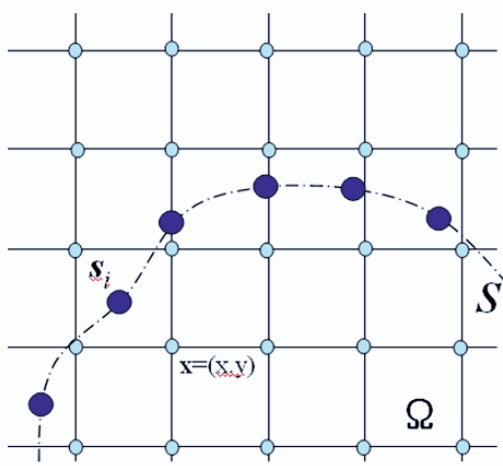

(a)

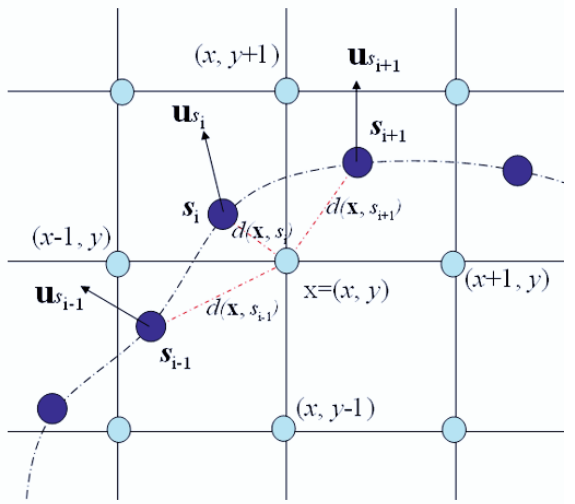

(b)

Fig. 1. (a) Schematic 2D representation of surface vertices overlaid on a discrete and rectangular control lattice $\boldsymbol{\Omega}$. (b) a vector $\mathbf{u}_{\mathbf{x}}$, as a boundary condition, is interpolated from displacement vectors on surface vertices $\left(\mathbf{u}_{\mathbf{s i - 1}}, \mathbf{u}_{\mathbf{s i}}, \mathbf{u}_{\mathbf{s i + 1}}\right)$ adjacent to $\mathbf{x}$.

Under the boundary condition determined in (4), the heat equation on the discrete domain $\boldsymbol{\Omega}$ is defined as:

$$
\begin{aligned}
& \mathbf{u}_{\mathbf{x}}(t)-\mathbf{u}_{\mathbf{x}}(t-1)=k \Delta \mathbf{u}_{\mathbf{x}}(t-1) \\
& =\frac{1}{6}(\mathbf{u}(x-1, y, z, t-1)+\mathbf{u}(x+1, y, z, t-1)+ \\
& \quad \mathbf{u}(x, y-1, z, t-1)+\mathbf{u}(x, y+1, z, t-1)+ \\
& \quad \mathbf{u}(x, y, z-1, t-1)+\mathbf{u}(x, y, z+1, t-1)-6 \mathbf{u}(x, y, z, t-1))
\end{aligned}
$$

$\mathbf{u}_{\mathbf{x}}(\mathrm{t})$ is updated by adding $\mathbf{u}_{\mathbf{x}}(\mathrm{t}-1)$ in (5).

The final displacement vector fields on $\mathbf{\Omega}$ are determined when:

$$
\sum_{\Omega}\left\|\mathbf{u}_{\mathbf{x}}(\mathrm{t})-\mathbf{u}_{\mathbf{x}}(\mathrm{t}-1)\right\|<\alpha
$$

In (6), the optimization threshold $\alpha$ was chosen to be 0.001 .

\subsection{Assessment of Local Volume Changes}

Local volume changes are quantified by the Jacobian determinant $\mathrm{J}_{S}[5]$ on surface points $S$, being defined as:

$$
J_{S}=\operatorname{det}\left(\mathrm{I}+\nabla \mathbf{u}_{S}\right)=1+\operatorname{tr}\left(\nabla \mathbf{u}_{S}\right)+\operatorname{det}_{2}\left(\nabla \mathbf{u}_{S}\right)+\operatorname{det}\left(\nabla \mathbf{u}_{S}\right)
$$

where $t r$ is the trace operator, $\operatorname{det}_{2}\left(\nabla \mathbf{u}_{S}\right)$ is the sum of $2 \times 2$ principal minors of $\nabla \mathbf{u}$.

For the vector fields produced by an isotropic diffusion, which assumes no shear deformation, the higher order terms approach zero. Therefore, we can approximate $J$ $\approx \operatorname{tr}\left(\nabla \mathbf{u}_{S}\right)$ when 1 is subtracted. This Jacobian explains growth or shrinkage of a unit- 
size cube through each of the 3 orthogonal axes. For surface-based analysis, we are only interested in assessing surface-normal directional changes. Therefore, the Jacobian at a surface point $s_{i}$ is simplified as:

$$
J_{s i}=\nabla \mathbf{u}_{s i} \cdot \mathbf{n}_{s i}=\left(\mathbf{u}_{s i}-\mathbf{u}_{s i}\right) \cdot \mathbf{n}_{s i}
$$

In equation $(\mathbf{8}), \mathbf{n}$ is the unit surface normal vector and $\hat{s}_{i}$ is the point where the distance from $s_{i}$ is 1 and its direction is the inward surface normal. Using tri-linear interpolation, $\mathbf{u}_{s i}$ and $\mathbf{u}_{s i}$ are determined from the displacement fields on $\boldsymbol{\Omega}$.

\section{Experiments and Results}

\subsection{Simulation Data}

We created a rounded cylindrical volume with a resolution of $0.25 \times 0.25 \times 0.25 \mathrm{~mm}^{3}$ (Figure 2-a) and applied the following deformations: bending (Figure 2-b, row 1), local shifting (Figure 2-b, row 2) and simultaneous local shrinking $(0.5 \mathrm{~mm})$ and shifting $(2.0 \mathrm{~cm}$ ) in z-axis (Figure 2-b, row 3). To compare the deformed object to the original, we first extracted SPHARM-PDM surfaces and calculated the displacement vectors. Then, at each vertex, we computed a signed surface-normal component of the displacement vector (SNV) and a surface-based Jacobian determinant (SJD). 3D mapping of the SNV showed mirrored inward/outward deformations on the two facing surfaces reflecting bending and shifting (Figure 2-d row 1, 2). However, in the case of simultaneous local shrinking and shifting, SNV did not allow differentiating shrinkage from shifting because of a stronger effect of shifting (Figure 2-d, row 3). Contrary to SNV, the SJD was not influenced by bending and shifting (Figure 2-e, row 1,2) and accurately localized the shrinkage (Figure 2-e, row 3).

To investigate the influence of the heat equation interpolation kernel size in detecting volume changes, we measured SJD with respect to different grid sizes of the 3D domain $\Omega$ ranging from $0.125 \times 0.125 \times 0.125 \mathrm{~mm}^{3}$ to $4.0 \times 4.0 \times 4.0 \mathrm{~mm}^{3}$. For this experiment, SJD was computed from the displacement vectors between the reference object and its variant deformed by simultaneous local shifting and shrinkage. We defined the ROI as the vertices on which we modeled the shrinkage. Then, for each grid size, we measured the mean SJD in the ROI and a modified standard error (MSE) over the entire object. MSE was defined as:

$$
M S E=\sum_{R O I}|S J D-(-0.5)| / n_{R O I}+\sum_{\underline{R O I}}|S J D| / n_{\underline{R O I}}
$$

In equation (9), $\mathrm{n}_{\mathrm{ROI}}$ is the number of vertices in the $R O I$ and $R O I$ defines the vertices outside the ROI. Mean SJD in the ROI showed that the target volume shrinkage was detected with a grid size ranging from $0.25 \times 0.25 \times 0.25$ (mean SJD: 0.53 ) to $0.5 \times 0.5 \times 0.5 \mathrm{~mm}^{3}$ (mean SJD: 0.49 ) (Figure 3-a). The MSE demonstrated that the minimum error is obtained at the grid size of $0.5 \times 0.5 \times 0.5 \mathrm{~mm}^{3}$ (Figure 3-b). 


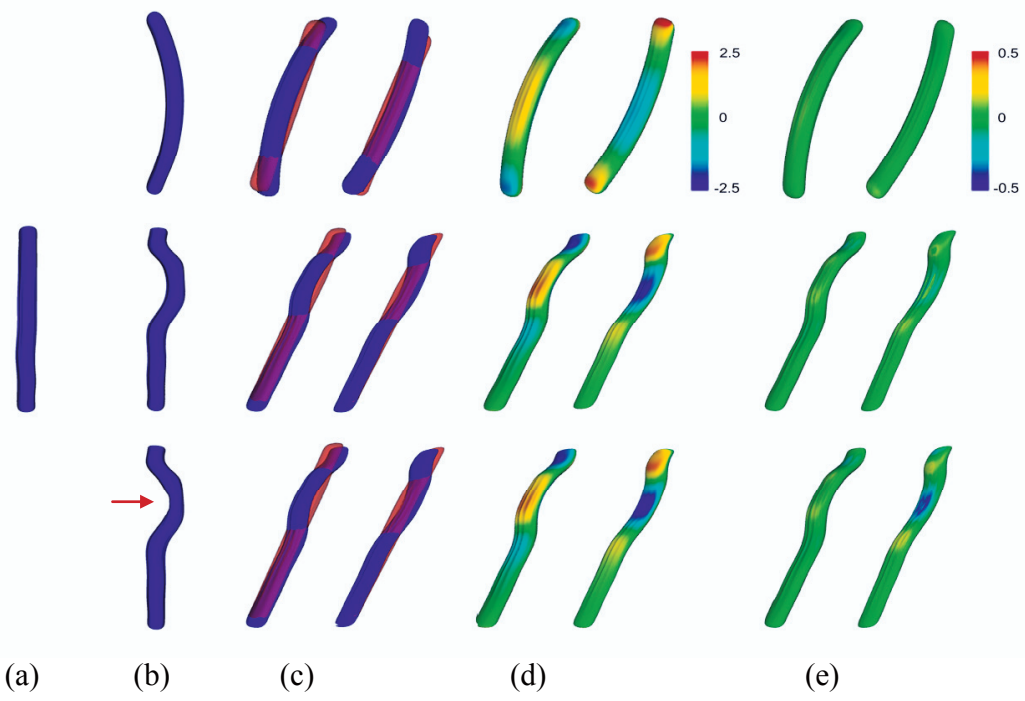

Fig. 2. Simulation. (a) Reference object. (b) Three deformed variants of the reference object: bending (top), local shifting (middle), simultaneous local shifting and shrinkage (bottom). The red arrow indicates the region where the local shrinking was applied. (c) Deformed objects (solid blue) aligned to the reference (transparent red). (d) Signed surface-normal components of the displacement vectors measured between the deformed objects and the reference (color scale in $\mathrm{mm},-/+$ signs indicate inward/outward deformation) (e) Surface-based Jacobian determinant maps (color scale in $\mathrm{mm}^{3},-/+$ signs indicate volume loss/growth).

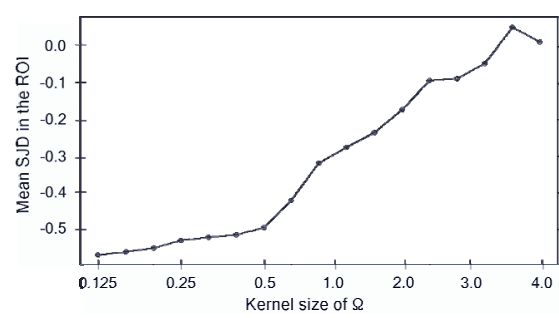

(a)

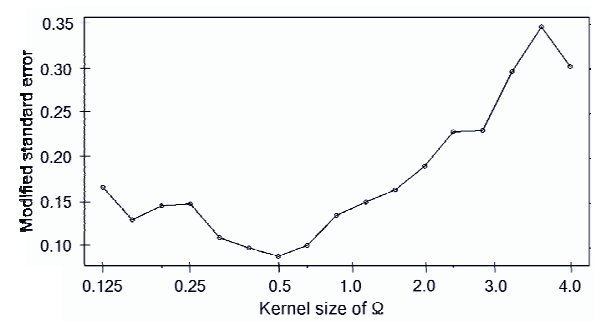

(b)

Fig. 3. Influence of the kernel size of the heat equation interpolation on the detection of $0.5 \mathrm{~mm}$ shrinkage. Graph of mean SJD in the ROI (a) and modified standard error over the entire object (b) across blurring kernels.

\subsection{Shape Analysis of the Hippocampus in Temporal Lobe Epilepsy}

Temporal lobe epilepsy (TLE) is associated with hippocampal sclerosis, a pathology consisting of various degrees of neuronal loss throughout the hippocampus. The most conspicuous MRI feature of hippocampal sclerosis is atrophy.

We selected from our database 22 medically intractable TLE patients $(11$ males; mean age: $37 \pm 11$ ) with a left-sided seizure focus and 33 age- and sex-matched healthy controls. Based on a 2SD cutoff from the mean volume of healthy controls, all 
patients had unilateral hippocampal atrophy. Group differences were assessed by vertex-wise t-tests with correction for multiple comparisons using FDR.

In patients, SNV detected areas of inward deformations at the level of hippocampal head and body ipsilateral to the seizure focus, and areas of mirrored inward/outward deformations in the tail due to local shifting (Figure 4-a). There were also areas of subtle focal inward deformation at the level of the contralateral hippocampal body.

SJD analysis revealed areas of atrophy that overlapped with inward deformation regions seen on the SNV map. In addition, SJD revealed volume loss at the level of hippocampal tail where SNV had detected mirrored inward/outward deformation. The extent of ipsi- and contralateral atrophy in the SJD maps was more widespread than in SNV. Representative examples are shown in four patients in Figure 4.

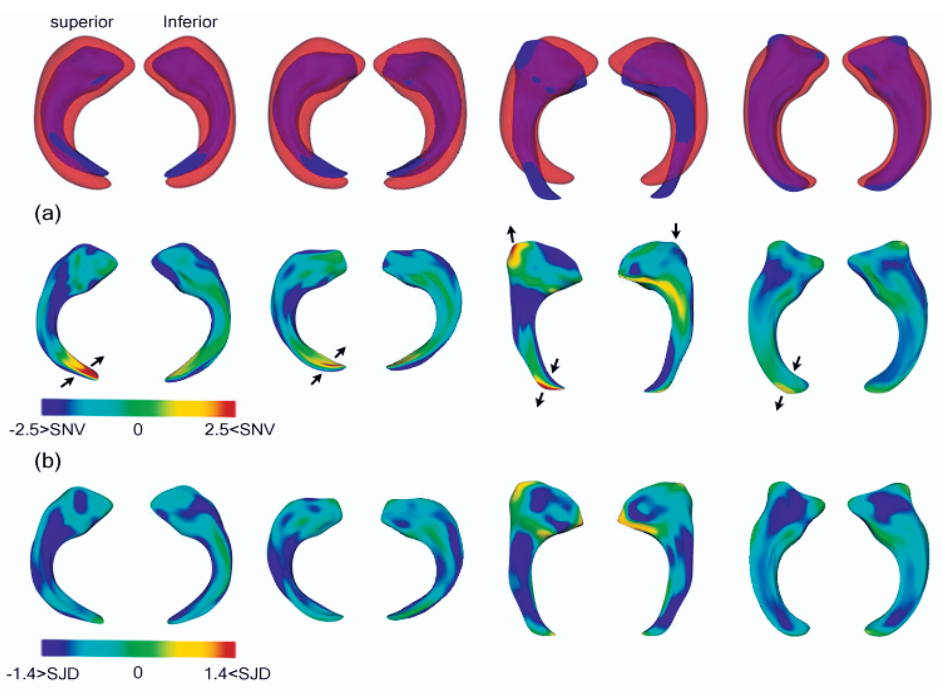

(c)

Fig. 4. Analysis of the left hippocampus in left TLE. (a) Four hippocampi (superior and inferior views) of TLE patients aligned to a template built from 33 control subjects. The template is shown in transparent red. The patients' hippocampi are in solid blue (purple shows areas of overlap with the template). (b) Signed surface-normal component maps of the displacement vectors (SNV; color scale in mm, -/+ signs indicate inward/outward deformation). Paired black arrows indicate the region where a local shifting/bending is detected. These changes at the level of the hippocampal tail are clearly visible in (a). (c) Surface-based Jacobian determinant maps (SJD; color scale in $\mathrm{mm}^{3}$, -/+ signs indicate volume loss/growth).

\section{Discussion and Conclusion}

Medial surface and axis models allow assessing local volume changes [3]. However, these thickness-based approaches are limited to the detection of symmetric alterations [3]. Diseased-related morphological changes, nevertheless, may result in asymmetrical deformations. While SPHARM-PDM accurately localizes asymmetrical morphological changes it does not yield insights in the nature of the difference, i.e., whether it is due to shrinkage or a shifting/bending process. 
We propose a new approach to differentiate these processes by computing the Jacobian determinant on the deformation field estimated from the displacement vectors produced by SPHARM. This is done by solving the heat equation using vertex-wise displacement vectors as a boundary condition. Our method enables the detection of local asymmetrical volume changes independent of shifting and bending, thus complementing SPHARM and providing new insights to the interpretation of morphological processes associated with neurological disorders.

Our experiments suggest that the heat equation interpolation kernel size should be equal or smaller than the expected volume changes, even though a too small kernel would decrease accuracy.

In a previous surface-based displacement analysis in TLE, the nature of posterior hippocampal deformations could not be interpreted [1]. In our study combining traditional SPHARM-PDM and surface-based Jacobian determinant, we were able to differentiate atrophy from displacement. In addition, surface-based Jacobian determinant allowed detecting atrophy where SPHARM showed mirrored changes.

In the current study, we adopted a template-dependent method to analyze the surface normal component of displacement vectors. Recently Styner et al. [4] developed a template free method to assess positioning changes. However, this method does not assess the direction of changes. Since our aim was to provide a biological meaningful interpretation of local volume changes, we favored a templatedependent approach. By creating a template made of a large number of hippocampal surfaces any bias related to shape was minimized.

\section{References}

1. Hogan, R.E., et al.: MRI-based high-dimensional hippocampal mapping in mesial temporal lobe epilepsy. Brain 127, 1731-1740 (2004)

2. Csernansky, J.G., et al.: Early DAT is distinguished from aging by high-dimensional mapping of the hippocampus. Neurology 55, 1636-1643 (2000)

3. Styner, M., et al.: Boundary and medial shape analysis of the hippocampus in schizophrenia. Medical Image Analysis 8, 197-203 (2004)

4. Styner, M., et al.: Statistical Shape Analysis of Brain Structures using SPHARM-PDM. In: MICCAI 2006 Opensource workshop (2006)

5. Chung, M.K., et al.: A unified statistical approach to deformation-based morphometry. NeuroImage 14, 595-606 (2001)

6. Ashburner, J., Friston, K.J.: Voxel-based morphometry: the methods. NeuroImage 11, 805$821(2000)$

7. Janke, A.L., et al.: 4D deformation modeling of cortical disease progression in Alzheimer's dementia. Magnetic Resonance in Medicine 46, 661-666 (2001)

8. Douglas Jr., J., Rachford Jr., H.H.: On the numerical solution of heat conduction problems in two and three space variables. Trans. Amer. Math. Soc. 82, 421-439 (1956) 